\title{
LA CONTAMINACIÓN ACÚSTICA, FACTOR MEDIO AMBIENTAL QUE INCIDE EN LA CALIDAD DE VIDA
}

\author{
NOISE POLLUTION, ENVIRONMENTAL FACTOR THAT SHINES IN THE \\ QUALITY OF LIFE
}

\author{
Jorge Lozano Cervera ${ }^{1}$; Rosa Requelme Ibañez ${ }^{2}$; Luis López Puycan ${ }^{3}$
}

\begin{abstract}
RESUMEN
El ruido es el contaminante más común y puede definirse como cualquier sonido que sea calificado, por quien lo percibe, como algo molesto, indeseado, inoportuno, perturbador o desagradable. De esta forma, lo que es música para una persona, puede ser considerado como ruido por otra. En este sentido, si bien la contaminación acústica es causada por el ruido procedente de diferentes fuentes, no ocasiona directamente enfermedades graves que se noten de inmediato, salvo en casos extremos como explosiones o ruidos de gran potencia. La contaminación acústica va causando poco a poco lesiones a la capacidad auditiva y daños a la salud mental de las personas expuestas.
\end{abstract}

Los principales efectos del ruido se han considerado como auditivos y no auditivos; los efectos auditivos están en correlación a la pérdida de la capacidad auditiva de las personas expuestas y los no auditivos son los que pueden generar estrés por perturbar el sueño, por ser transformadores de las actividades humanas cotidianas o por efectos en el comportamiento humano.

Palabras clave: Ruido, contaminación acústica, decibelio, factor medioambiental, sonómetro.

\begin{abstract}
Noise is the most common contaminant and can be defined as any sound that is qualified by the perceiver, as intrusive, unwanted, unwelcome, disruptive or unpleasant. In this way, which is music to a person can be considered as noise on the other. In this sense, although noise pollution is caused by noise from different sources, not directly causes serious diseases are noticed immediately, except in extreme cases such as explosions or powerful noises. Noise pollution is gradually causing hearing injury and damage to the mental healt of the people exposed.

The main effects of noise have been considered as auditory and non-auditory, auditory effects are correlated to the loss of hearing of those exposed and not hearing are those that can generate stress disrupt sleep, being transformers everyday human activities or effects on human behavior.
\end{abstract}

Keywords: Noise, noise, decibel, environmental factor, sound level meter.

\section{INTRODUCCIÓN}

La contaminación acústica es considerada por la población como un factor medioambiental muy importante, que incide de forma principal en su calidad de vida.

La contaminación ambiental urbana o ruido ambiental es consecuencia directa de las actividades que se desarrollan en las grandes ciudades.

El término contaminación acústica hace referencia al ruido cuando éste se considera un contaminante, es decir, sonido que puede producir efectos fisiológicos y psicológicos nocivos para una persona o grupo de personas.

La causa principal de la contaminación acústica es la actividad humana. Tenemos el transporte, la construcción de edificios y obras públicas, la industria, entre otras.

Los efectos producidos por el ruido pueden ser fisiológicos, como la pérdida de audición, y psicológicos, como la irritabilidad exagerada.

El ruido se mide en decibelios $(\mathrm{dB})$. Los equipos de medida más utilizados son los sonómetros. Un

\footnotetext{
'Licenciado en Matemáticas, Magíster en Computación e Informática. Facultad de Ciencias de la UNJBG.

'Licenciado en Matemáticas, Magíster en Docencia Universitaria. Facultad de Ciencias de la UNJBG.

${ }^{3}$ Licenciado en Estadística. Facultad de Ciencias de la UNJBG.
} 
informe de la Organización Mundial de la Salud (OMS), considera $50 \mathrm{~dB}$ como el límite superior deseable.

Técnicamente, el ruido es un tipo de energía secundaria de los procesos o actividades que se propaga en el ambiente, en forma de ondulatoria compleja, desde el foco productor, hasta el receptor, a una velocidad determinada y disminuyendo su intensidad con la distancia y el entorno físico.

La contaminación acústica perturba las distintas actividades comunitarias, interfiriendo la comunicación hablada, base de la convivencia humana. Altera el sueño, el descanso y la relajación, impidiendo la concentración y el aprendizaje, y lo que es más grave, crea estados de cansancio y tensión que pueden degenerar en enfermedades de tipo nervioso y cardiovascular.

Existe documentación sobre las molestias de los ruidos en las ciudades, desde la antigüedad, pero es a partir del siglo pasado, como consecuencia de la evolución industrial, del desarrollo de nuevos medios de transporte y del crecimiento de las ciudades, que aparece realmente el problema de la contaminación acústica urbana.

Dos son las causas fundamentales: el aumento espectacular del parque automotor en los últimos años y las ciudades no han sido diseñadas para soportar los medios de transporte.

\section{MATERIALES Y MÉTODOS}

\subsection{Materiales}

Las mediciones se realizaron con un medidor de nivel sonoro o decibelímetro (Digital Sound level meter EXTECH 407736), y el Sistema de Posición Global (GPS MAP76CSx), registrándose así los valores ambiente y los picos sonoros.

Para determinar el modelo matemático del ambiente sonoro se utilizó el software Matlab7.

\subsection{Metodología}

\subsubsection{Línea de base}

- Durante el día se realizó una campaña de Línea de Base de ruido, en el Distrito Coronel Gregorio Albarracín Lanchipa de Tacna.

- Se consideró como muestra una de las rutas más transitadas que conecta dicho distrito con el centro de la ciudad de Tacna.

- Para alcanzar los objetivos propuestos se realizó, en primera instancia, un monitoreo a lo largo de 60 minutos $\mathrm{y}$ en distintos momentos del día (mañana y tarde).

- El sonómetro se ubicó a una altura de 1,5 metros en relación al suelo y, de 1,5 a 2 metros de distancia de los vehículos.

- Los sectores donde se realizaron la toma de datos son calles y avenidas que están asfaltadas: Ovalo Cuzco, avenida Municipal, Mercado Santa Rosa, Ovalo la cultura y colegio Gerardo Arias Copaja.

\subsubsection{Modelación del ambiente sonoro}

La variable de entrada del modelo se refiere a las potencias sonoras de las fuentes de ruido para cada etapa contemplada. Decibel $(\mathrm{dB})$ es la unidad de nivel sonoro medido con un filtro previo que quita parte de las bajas y las muy altas frecuencias.

El decibel es una proporción sin dimensiones distribuida en un intervalo logarítmico. El valor más bajo $(\mathrm{O} \mathrm{dB})$ corresponde al umbral de audición, mientras que el nivel más alto, teóricamente ilimitado, se establece en el umbral del dolor (140 $\mathrm{dB})$. Es una variable cuantitativa continua.

El software de simulación computacional utilizado corresponde a ArcGis 10, el cual incorpora todas las variables físicas de topografía y características acústicas de las fuentes sonoras.

La temperatura se fijó en $15^{\circ} \mathrm{C}$ y la humedad relativa en $75 \%$, constituyendo un escenario desfavorable por la baja atenuación de la propagación de la onda sonora, debido a estos efectos meteorológicos.

Además, la norma de cálculo utilizada considera siempre la velocidad del viento entre 3 y $4(\mathrm{~m} / \mathrm{s})$ a favor de la propagación. 


\subsubsection{Puntos de Medición}

Tabla $\mathrm{N}^{\circ}$ 01: Puntos de medición.

\begin{tabular}{|c|c|c|c|}
\hline $\mathrm{N}^{\circ}$ & $\begin{array}{c}\text { Motor } \\
\text { decibeles }\end{array}$ & GPS & Ubicación \\
\hline 1 & 83.1 & 169 & Ovalo Cuzco \\
\hline 2 & 89.9 & 170 & $\begin{array}{l}\text { Mercado Héroes del } \\
\text { Cenepa }\end{array}$ \\
\hline 3 & 92.6 & 171 & $\begin{array}{l}\text { Plaza Pérez Gamboa } \\
\text { (semáforo) }\end{array}$ \\
\hline 4 & 87.3 & 172 & $\begin{array}{l}\text { Municipalidad GAL } \\
\text { (Semáforo) }\end{array}$ \\
\hline 5 & 85.4 & 173 & $\begin{array}{l}\text { Grifo la Esperanza } \\
\text { "PECSA" }\end{array}$ \\
\hline 6 & 89.0 & 174 & Mercado Santa Rosa \\
\hline 7 & 79.1 & 175 & $\begin{array}{l}\text { Costado del Mercado. Sta. } \\
\text { Rosa }\end{array}$ \\
\hline 8 & 91.8 & 176 & Ovalo la Cultura \\
\hline 9 & 87.6 & 177 & $\begin{array}{l}\text { Colegio Gerardo Arias } \\
\text { Copaja }\end{array}$ \\
\hline 10 & 87.8 & 178 & $\begin{array}{l}\text { Frente al Colegio Gerardo } \\
\text { Arias Copaja }\end{array}$ \\
\hline 11 & 87.9 & 179 & $\begin{array}{l}\text { Frente al Mercado. Sta. } \\
\text { Rosa }\end{array}$ \\
\hline 12 & 86.6 & 180 & $\begin{array}{l}\text { Frente Costado del } \\
\text { Mercado. Sta. Rosa (Banco } \\
\text { Azteca) }\end{array}$ \\
\hline 13 & 85.9 & 181 & $\begin{array}{l}\text { Frente al Grifo la } \\
\text { Esperanza } \\
\text { "PECSA" }\end{array}$ \\
\hline 14 & 86.8 & 182 & $\begin{array}{l}\text { Frente a la Municipalidad } \\
\text { GAL (Semáforo) }\end{array}$ \\
\hline 15 & 86.9 & 183 & $\begin{array}{l}\text { Frente a Plaza Pérez } \\
\text { Gamboa (semáforo) }\end{array}$ \\
\hline 16 & 91.5 & 184 & $\begin{array}{l}\text { Frente al Mercado. Héroes } \\
\text { del Cenepa }\end{array}$ \\
\hline 17 & 87.2 & 185 & Frente al Ovalo Cuzco \\
\hline 18 & 72.9 & 186 & $\begin{array}{l}\text { Av. Humboldt (grifo } \\
\text { satélite del sur) }\end{array}$ \\
\hline
\end{tabular}

Fuente: Propia

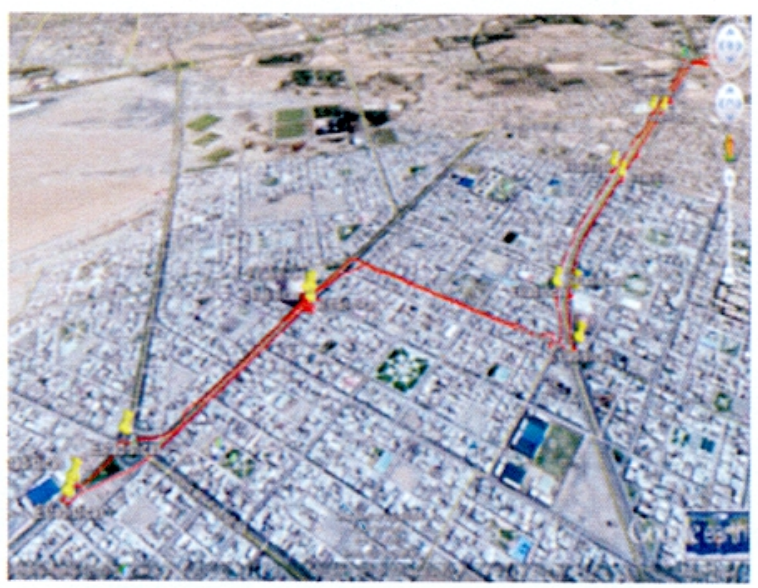

Gráfico $\mathrm{N}^{\circ} 01$ : Trayectoria de la medición.

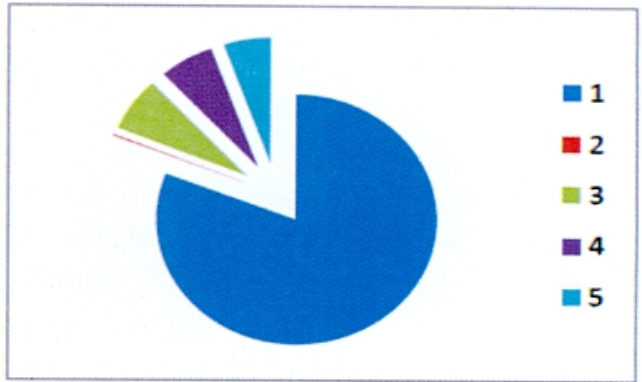

Gráfico $\mathrm{N}^{\circ}$ o2: Fuentes de contaminación acústica en el Distrito Coronel Gregorio Albarracín Lanchipa.

Donde:

1. Tráfico terrestre, 2. Fuentes de origen animal, 3. Fuentes de origen comunitario, 4. Fuentes de origen mecánico, 5. Actividades comerciales y de ocio.

Se aprecia claramente que la principal fuente de emisión de ruidos es el tráfico terrestre ( $81 \%)$, es decir, el trasiego de turismo, motocicletas, vehículos pesados y demás. Luego se encuentran otro tipo de fuentes menos importantes de origen comunitario, mecánico, actividades de ocio y comerciales. En casos puntuales éstas últimas pueden llegar a ser bastante representativas como en las zonas anexas al mercado Santa Rosa.

En el siguiente gráfico se reflejan las aportaciones porcentuales de las diferentes fuentes de tráfico terrestre (automóviles, motocicletas, camiones, camiones de recogida de basura, ambulancias y sirenas, etc.).

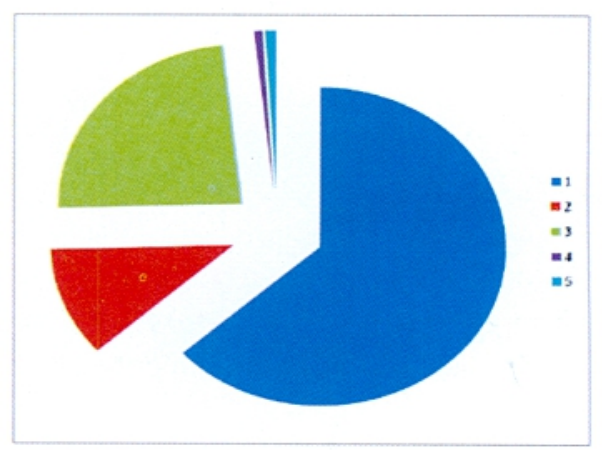

Gráfico $\mathrm{N}^{\circ}$ 03: Fuentes de contaminación acústica incluidas en el tráfico terrestre.

Donde:

1. Automóviles y turismo, 2. Camiones y autocares, 3 . Motos y motocicletas, 4. Camiones de basura, 5 . Ambulancias y sirenas.

Según el gráfico anterior, los automóviles, las líneas de turismo, así como las motos y motocicletas, son las principales causas de 
contaminación acústica por tráfico terrestre y, por ende, las principales fuentes generadoras de la emisión de ruidos en el Distrito G.A.L.

\section{RESULTADOS}

La tabla $\mathrm{N}^{\circ} \mathrm{O} 2$ muestra los resultados estadísticos sobre la contaminación acústica tomados desde el inicio del óvalo Cuzco y culminando en el Colegio Gerardo Arias Copaja en el distrito Gregorio Albarracín Lanchipa-2012.

Tabla $\mathrm{N}^{\circ} \mathbf{0 2}$ : Resuitados estadísticos.

\begin{tabular}{|l|l|}
\hline $\mathbf{N}^{\circ}$ de Datos Válidos: 19 & Decibeles (motor) \\
\hline Media & 85,7105 \\
\hline Mediana & 85,5000 \\
\hline Moda & 85,50 \\
\hline Desviación típica & 3,34646 \\
\hline Rango & 14,00 \\
\hline Mínimo & 76,40 \\
\hline Máximo & 90,40 \\
\hline
\end{tabular}

Fuente: Propia

\section{Modelamiento del ambiente sonoro}

Para obtener los datos del modelo matemático, que represente la intensidad de sonido en decibeles, fue necesario obtener las distancias en metros, entre cada uno de los puntos de muestra. Se eligieron solo los datos (09) del primer recorrido (ida), y con estas distancias acumuladas se obtuvieron los datos que se muestran en la Tabla $\mathrm{N}^{\circ} 03$ :

Tabla $\mathrm{N}^{\circ}$ 03: Distancia entre las mediciones.

\begin{tabular}{|l|l|c|c|c|}
\hline$N^{\circ}$ & \multicolumn{1}{|c|}{ Ubicación } & $\begin{array}{r}\text { Distancia } \\
\text { de punto } \\
\text { a punto }\end{array}$ & $\begin{array}{c}\text { Distancia } \\
\text { acumulada }\end{array}$ & decibeles \\
\hline 1 & Ovalo Cuzco & 0 & 0 & 79.0 \\
\hline 2 & Mcd. Cenepa & 555 & 555 & 82.0 \\
\hline 3 & Plz. Pérez G. & 482 & 1037 & 73.5 \\
\hline 4 & Munc. GAL & 622 & 1659 & 76.7 \\
\hline 5 & Grifo la Esp. & 191 & 1850 & 73.8 \\
\hline 6 & Mcd. Sta. Rosa & 633 & 2483 & 78.6 \\
\hline 7 & $\begin{array}{l}\text { Costado. Mcd. Sta. } \\
\text { Rosa }\end{array}$ & 11 & 2494 & 69.6 \\
\hline 8 & Ovalo la cultura & 493 & 2987 & 65.9 \\
\hline 9 & Colegio Arias Copaja & 112 & 3099 & 67.4 \\
\hline
\end{tabular}

Fuente: Propia

\section{Obtención del modelo matemático}

En este caso, se utiliza la regresión polinomial. El polinomio de grado " $\mathrm{n}$ ", en general, tiene la forma:

$$
P(x)=a_{0}+a_{1} x+a_{2} x^{2}+a_{3} x^{3}+\cdots+a_{n} x^{n}
$$

Se realizó diferentes pruebas con respecto a los grados, hasta elegir el correcto, teniendo en cuenta la teoría sobre las intensidades de los sonidos. Con ese fin fue creado un programa codificado en el lenguaje de programación MATLAB versión 10.

A continuación, analizamos con el programa mencionado, los polinomios de grado 4, de grado 7 y de grado 8 .

\section{Análisis con un polinomio de grado 4}

El análisis se realizó con los datos del campo distancia acumulada versus oído a decibeles.

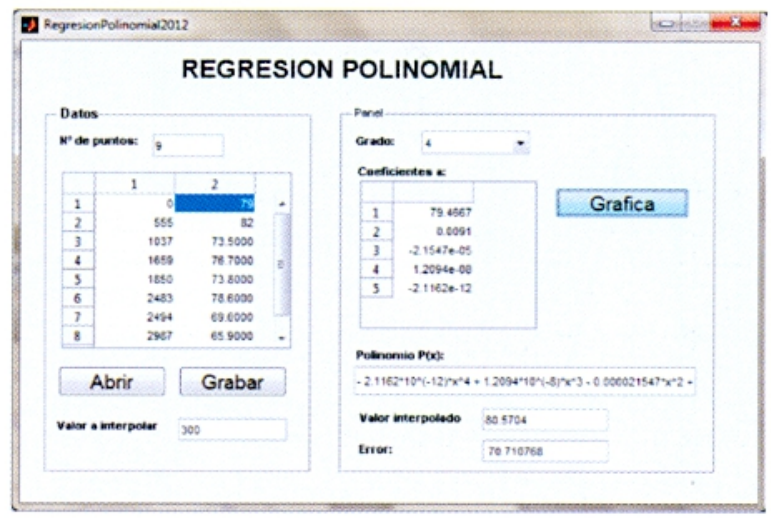

Gráfico $\mathrm{N}^{\circ}$ 04: Regresión polinomial grado 4 .

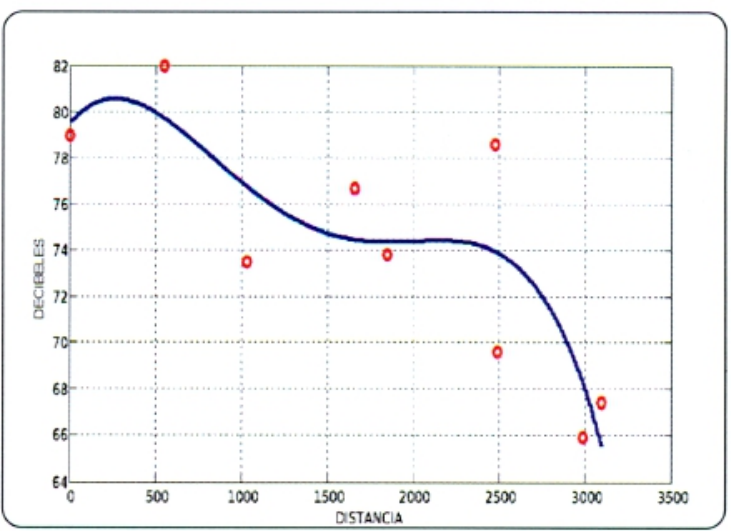

Gráfico $\mathbf{N}^{\circ}$ 05: Regresión polinomial construido con los datos del campo distancia acumulada versus los datos del campo oído a decibeles.

\section{Análisis con un polinomio de grado 7}

Se procedió con los datos del campo distancia acumulada versus los datos del campo oído a decibeles. 


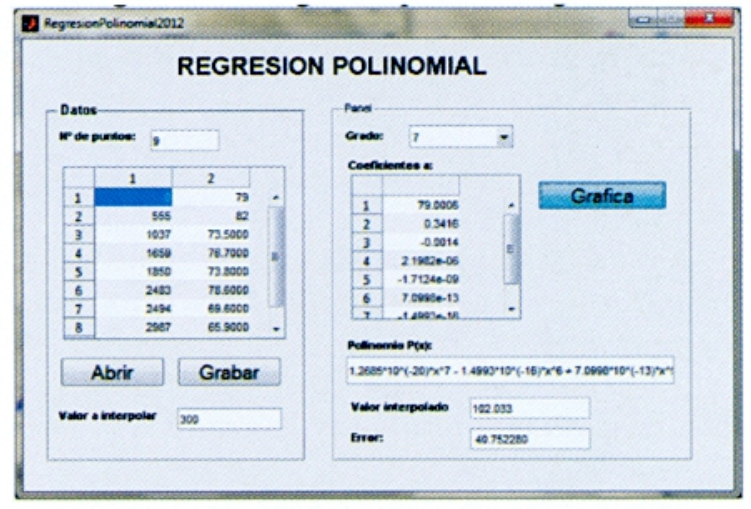

Gráfico $\mathrm{N}^{\circ}$ 06: Regresión polinomial grado 7.

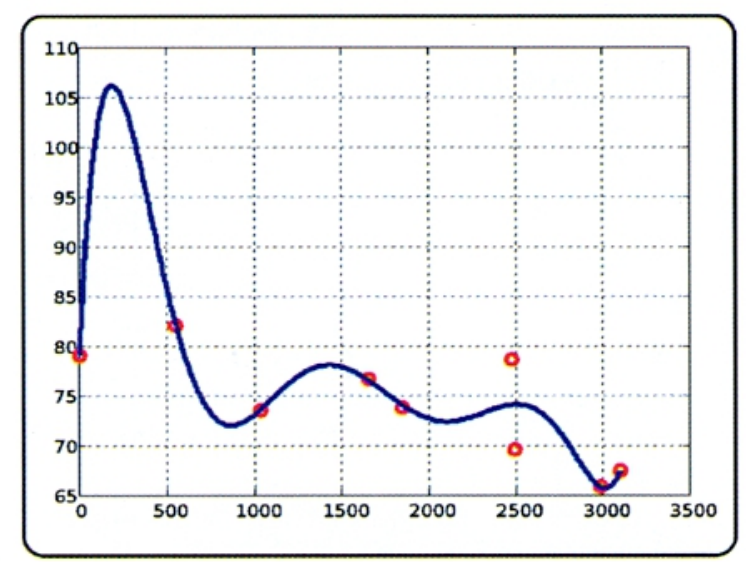

Gráfico $\mathrm{N}^{\circ} \mathbf{0 7}$ : La regresión polinomial grado 4 se ha construido con los datos del campo distancia acumulada versus los datos del campo oído a decibeles.

Por lo anteriormente mostrado, y porque el sonido que se da entre los puntos de medición deben ser los menores que los otros, se eligió el polinomio de cuarto grado.

$$
\begin{aligned}
\mathrm{P}(\mathrm{x})= & -2.1162 * 10-12 \times 4+1.2094 * 10-8 \times 3- \\
& 0.000021547 \times 2+0.009112 \mathrm{x}+ \\
& 79.467
\end{aligned}
$$

Este polinomio representa el modelo matemático que, además, permite hallar valores para otros puntos que no se tomaron en cuenta, por ejemplo, si un punto se elige a unos 300 metros del óvalo, la intensidad de sonido sería 80.5074.

\section{EVALUACIÓN DE RESULTADOS}

Tabla $\mathbf{N}^{\circ}$ 03: Estándares nacionales de calidad ambiental del ruido.

\begin{tabular}{|c|c|c|}
\hline \multirow{2}{*}{$\begin{array}{c}\text { Zona de } \\
\text { Aplicación }\end{array}$} & \multicolumn{2}{|c|}{ Valores expresado en Leq } \\
\cline { 2 - 3 } & $\begin{array}{c}\text { Horario } \\
\text { diurno }\end{array}$ & $\begin{array}{c}\text { Horario } \\
\text { nocturno }\end{array}$ \\
\hline $\begin{array}{c}\text { Zona de } \\
\text { Protección } \\
\text { Especial }\end{array}$ & $50 \mathrm{~dB}$ & $40 \mathrm{~dB}$ \\
\hline Zona Residencial & $60 \mathrm{~dB}$ & $50 \mathrm{~dB}$ \\
\hline Zona Comercial & $70 \mathrm{~dB}$ & $60 \mathrm{~dB}$ \\
\hline Zona Industrial & $80 \mathrm{~dB}$ & $70 \mathrm{~dB}$ \\
\hline
\end{tabular}

Fuente: Propia

\section{Comparación con otros resultados}

- Los niveles de ruido encontrados en el distrito de G. A. L., el valor estándar establecido para zonas mixtas (residencial-comercial D. S. $\mathrm{N}^{\circ}$ 085-2003-PCM), deben ser menor a $60 \mathrm{~dB}$.

- Los niveles de ruido obtenidos durante el monitoreo en 18 puntos en el distrito de G. A. L. se encuentran entre $61.2 \mathrm{~dB}$ y $82 \mathrm{~dB}$.

- Se observa que el lugar en donde se registra la mayor contaminación sonora es en el mercado Héroes del Cenepa con 82.0 dB.

- Y que el lugar en donde se registra la menor intensidad de ruido es en el ovalo la cultura con $65.9 \mathrm{~dB}$.

\section{CONCLUSIONES}

- En el presente trabajo de investigación, hemos obtenido dos mapas acústicos y nos muestra que el nivel sonoro es mayor de $65 \mathrm{~dB}$ en los diferentes sectores de la ruta estudiada.

- Los vehículos motorizados son la principal fuente de contaminación acústica en el Distrito Gregorio Albarracín Lanchipa superando los límites permisibles.

- En algunos sectores, debido a la diversidad y multiplicidad de fuentes, es complejo determinar cuál es la que produce mayor ruido acústico.

\section{RECOMENDACIONES}

\section{En el campo del conocimiento}

Utilizar equipos de alta precisión para obtener los datos de las diferentes fuentes de 
ruido y conocer en forma precisa sus niveles. Estos datos tienen que estar especificados en mapas acústicos suficientemente amplios y detallados para realizar el control respectivo.

\section{En el campo de las normas}

Las instituciones responsables deberán tomar todas las medidas para hacer cumplir las normas existentes en relación al ruido.

\section{En el campo de las acciones (Información- Formación)}

Tener en cuenta tres grupos de acciones:

1. Donde existen mapas de ruido, dar a conocer a la ciudadanía, mediante los medios de difusión.

2. Una buena parte del ruido que se genera en las ciudades depende del conductor del vehículo, por lo que se le debe informar sobre las consecuencias de la contaminación acústica.

3. La información y la formación sobre el ruido no tiene que reducirse a acciones puntuales, sino debe ir más allá y formar parte de los valores y de los hábitos de las personas. Se debe pasar de la cultura del ruido a la cultura del silencio, el cual debería ser un valor importante a alcanzar en nuestra sociedad.

El estudio realizado en el presente trabajo es importante porque contribuirá al mejoramiento de la calidad de vida de la ciudadanía del Distrito Gregorio Albarracín Lanchipa, si se le toma en cuenta

\section{Otras acciones:}

- Mantenimiento periódico del parque automotor.

- Actuar directamente sobre las fuentes de ruido (cuando se trata de equipamiento, a través de protectores acústicos materiales que absorben los ruidos), y cuando se trata de personas, a través de la educación (charlas formativas, cartelería y ajustes operativos).
- Actuar desde la arquitectura, aislando los locales que puedan tener prioridad en cuanto a la protección, utilizando elementos de sellado de rendijas (burletes, etc.).

- La municipalidad debe informar y sensibilizar a los vecinos albarracinos acerca de la contaminación acústica y de los daños que ocasionan a las personas que están expuestas al ruido y realizar una lucha frontal en contra de la contaminación acústica, con la campaña “ ¡No al ruido!"

\section{REFERENCIAS BIBLIOGRÁFICAS}

Benjamín García Sanz, Francisco Javier Garrido. La Contaminación Acústica en Nuestras Ciudades. Url:www.estudios.lacaixa.es.

Arizmendi, L. J. (1995). Contaminación acústica y urbanismo. Fundación Bancaja (ed.), La contaminación sonora: evaluación efectos y control. Valencia.

\section{REFERENCIAS ELECTRÓNICAS}

- http://www.proaudio.com.ve/contenido.php? articulo_no=21

-http://www.aytorota.es/doc/medio_amb iente/indicadores_ambientales/11.contamina cion_acustica.pdf

-http:// www.proaudio.com.ve

\section{Correspondencia:}

Jorge Lozano Cervera

Ciudad Universitaria fundo "Los Granados"

Av. Miraflores s/n Tacna - Perú

Rosa Requelme Ibañez

Ciudad Universitaria fundo "Los Granados"

Av. Miraflores s/n Tacna - Perú

Luis Lopez Puycan

Ciudad Universitaria fundo "Los Granados" Av. Miraflores s/n Tacna - Perú 